\title{
El libro antiguo y la reutilización de manuscritos: relevamiento y catalogación de fragmentos manuscritos in situ en colecciones argentinas 1
}

The ancient book and the reutilization of manuscripts: a survey and catalog of in situ manuscript fragments in Argentinian collections

\author{
Marcela Borelli \\ Instituto de Investigaciones Bibliográficas y Crítica Textual (IIBICRIT - CONICET), Argentina \\ Universidad de Buenos Aires, Argentina \\ Universidad Nacional de San Martín, Argentina \\ mborelli@uba.ar \\ iD https://orcid.org/0000-0001-9918-8776 \\ Olga Soledad Bobdziewicz \\ Instituto de Investigaciones Bibliográficas y Critica Textual ((IIBICRIT - CONICET), Argentina \\ Universidad de Buenos Aires, Argentina \\ soledad.bohdziewicz@conicet.gov.ar \\ iD https://orcid.org/0000-0002-7234-4377
}

\section{RESUMEN:}

Este trabajo se propone brindar una aproximación al antiguo libro impreso existente en nuestras colecciones nacionales a través del examen de un aspecto hasta ahora poco estudiado: la presencia de fragmentos de manuscritos medievales y humanísticos empleados como refuerzos de diversa índole en las encuadernaciones, reconociendo su tipología a través del análisis de casos representativos. Palabras clave: Fragmentología, Encuadernaciones, Manuscritos medievales, Manuscritos humanísticos, Libro Antiguo, Colecciones, Argentina.

\section{Abstract:}

The purpose of this paper is to offer an unexplored approach to the studies of the old printed books in Argentinian libraries: the presence of medieval and humanistic manuscript fragments used as binding material. We will offer a typology of $i n$ situ fragments according to their function through some exemplary cases.

KEYWORDs: Fragmentology, Bindings, Medieval manuscripts, Humanistic manuscripts, Ancient book, Argentina.

\section{INTRODUCCIÓN}

En las últimas décadas, la investigación sobre el patrimonio del libro antiguo en América Latina ha tomado especial impulso. A pesar de los reveses económicos que cualquier investigación sufre en los países emergentes, la construcción de catálogos que reúnan, unifiquen y pongan en conocimiento de la comunidad nuestro patrimonio sigue captando el interés y esfuerzo de los especialistas. Con todo, hay un aspecto del estudio del libro antiguo que hasta hace un par de años aún no había merecido una investigación sistemática: los fragmentos utilizados como refuerzos de distinto tipo para la encuadernación de libros, tanto manuscritos como impresos.

El proyecto "Fragmentos de Manuscritos en Argentina" del que formamos parte junto con especialistas de otras áreas, ha estado trabajando en la identificación, descripción, edición, catalogación y digitalización

Recepción: 07 Diciembre 2020 | Aceptación: 24 Febrero 2021| Publicación: 01 Octubre 2021 
de fragmentos manuscritos en el territorio nacional, lo que implica la colaboración de especialistas en las distintas áreas de los estudios medievales dada la diversidad de los textos estudiados: historia, musicología, filosofía, filología, etc.

Si bien en Argentina hubo intentos por ofrecer catálogos colectivos centrados en el patrimonio bibliográfico antiguo, como es el caso del impulsado por la Biblioteca Nacional hasta el año 2015 -Catálogo Nacional Unificado (CNU) de impresos editados antes de 1800 (Fernández Rojo, 2009)-, que tuvo el objetivo de relevar libros antiguos tanto en bibliotecas públicas como privadas, en general carecen de continuidad y, más aún, los resultados de esas búsquedas y catalogaciones no están a disposición para su consulta. Esto hace que al día de hoy sea extremadamente difícil estimar el número de libros antiguos en Argentina, ${ }^{3}$ cuáles son, o conocer en qué instituciones se conservan, su procedencia y fechas de impresión. Y cabe señalar que, más allá de los intereses concretos de algunas investigaciones, como la nuestra, que requieren de la localización de libros antiguos desde el siglo XV al XVII, esta falta de estudios y, por tanto, la escasa noticia que se tiene de su existencia, sumándose a la precariedad en el mantenimiento y custodia de este tipo de fondos, ofrece el clima ideal para que este rico patrimonio cultural se disperse, perdiéndose lenta e irremediablemente en muchos casos (Tesler, 2020. Por esta razón, la investigación y el trabajo permanente sobre este tipo de acervos, al darlos a conocer y destacar su valor, constituyen un aporte para su protección al ofrecer a quienes indagan estos materiales otra vía para su acceso. Ello contribuye de una manera virtuosa a resolver el conflicto que muchas veces existe entre la preservación de estos materiales frágiles - de cuya consulta se priva en ocasiones incluso a investigadores y especialistas más allá de las razonables disposiciones- y su estudio, aquello que las bibliotecas, por definición, tienen la misión de facilitar.

En líneas generales, podríamos decir que un fragmento es parte de un escrito mayor, fuera éste un libro en formato de códice o un documento, escrito en pergamino -y menos frecuentemente en papel o en papiroque es percibido como tal porque es el resultado de una mutilación producida por factores intencionales o accidentales. Todo fragmento posee una historia, un antes en el que fue creado para verse y ser de una manera completamente distinta a como se lo observa ahora. Entre este antes y después hubo un punto de inflexión que es considerado una instancia de no retorno (Molinari, Biondi y Abate, 2019).

Hemos comenzado refiriéndonos al libro antiguo porque la investigación sobre los fragmentos manuscritos está estrechamente ligada a su historia. En efecto, la mayor parte de los que hemos encontrado se encuentran in situ, es decir que están alojados en la encuadernación de libri tradentes, esto es, otros manuscritos, incunables y demás impresos tempranos. El conocimiento de este tipo de patrimonio en Argentina está todavía en ciernes. No ha existido hasta el momento un estudio sistemático al respecto y el nuestro es, hasta donde sabemos, el primer proyecto de esta naturaleza.

El origen de los fondos de libros antiguos sobre los que hemos trabajado o relevado como potenciales candidatos a contener fragmentos podría dividirse en dos tipos: aquellos que llegaron a partir del siglo XVI para abastecer las bibliotecas, escuelas y universidades de las órdenes religiosas; y los que forman o formaron parte de colecciones privadas adquiridas a lo largo de las primeras décadas del siglo XX. Hasta el momento hemos trabajado principalmente con estos últimos.

\section{Aspectos PRELIMINARES DE LA INVESTIGACión FRAGMENTOLÓGiCA}

En un gran número de casos, las bibliotecas, tanto de Europa como de países de América del Norte, poseen bibliotecas cuyos fondos antiguos dan lugar a la creación de proyectos basados exclusivamente en ellas. Un ejemplo emblemático es el de la Bodleian Library, que cuenta con una colección de alrededor de siete mil incunables, caso analizado por Mullett (2018). Nuestro proyecto se acerca a éste, ya que también se ocupa de fragmentos in situ, aunque ciertamente la situación es muy disímil desde un punto de vista cuantitativo. 
Decíamos que cuantitativamente nuestro caso se diferencia y lo hace de manera notable debido a la historia cultural y las vicisitudes de las instituciones, lo que hace que en la búsqueda de fragmentos debamos acudir a repositorios dispersos en nuestro amplio territorio nacional en pos de constituir un corpus de trabajo. Esto implica que tengamos que explorar de manera simultánea una cantidad de museos, archivos y bibliotecas para hacer la indagación, en primer lugar, acerca de las características de sus fondos. Dado el desafío que significa encarar este tipo de búsquedas, a efectos de su factibilidad, fue necesario, para esta primera etapa de nuestro proyecto, delimitar las áreas geográficas. Esto implicó tomar en consideración inicialmente la Ciudad Autónoma de Buenos Aires y Córdoba Capital, aunque en el futuro prevemos incluir otras ciudades o localidades.

Un aspecto que conspira contra la celeridad de las búsquedas es la falta de herramientas en línea o sus características, situación complejizada en algunas ocasiones por la falta incluso de catálogos físicos o inventarios para efectuar una primera aproximación antes de acudir a la inspección personal de los volúmenes. Estos últimos casos hacen particularmente dificultosas las búsquedas, ya que no es posible conocer de manera anticipada el tipo de fondos que se albergan. De hecho, por distintos motivos, hay un gran número de instituciones que carece de catalogaciones. En las instancias en que se cuenta con registros informatizados, a pesar de que ello nos permite conocer de manera remota de qué libros se componen las bibliotecas, en algunos casos no es posible efectuar búsquedas aplicando como criterio la datación, lo que es un aspecto que grosso modo, como hemos explicado más arriba, nos permite saber si se trata de volúmenes que puedan contener fragmentos. A esto debe agregarse que, incluso en aquellos casos en los que se cuenta con descripciones más exhaustivas, estas no suelen dar cuenta de su presencia dentro de las encuadernaciones, porque dependiendo de la época o de las prácticas de descripción adoptadas, podía ser un aspecto carente de relevancia, y que además requiere de una formación muy específica para ser analizado correctamente. Por su parte, en aquellos casos -que son, por cierto, los menos- en los que se da noticia de la existencia de fragmentos, la información que se proporciona de ellos es insuficiente de acuerdo con criterios paleográficos y codicológicos.

A partir de lo expuesto anteriormente, queda en evidencia la necesidad de establecer contacto con las autoridades y el personal a cargo de las instituciones para poner en su conocimiento el tipo de búsqueda que realizamos. De constatarse la existencia de libros antiguos, en un sentido bastante laxo, el siguiente paso es una inspección presencial de cada uno de los ejemplares que por su fecha de impresión están en el arco temporal principal. Ello de ninguna manera implica descartar apriorísticamente los libros menos antiguos, ya que una cantidad de piezas de interés ha sido hallada en la encuadernación de obras del siglo XX. ${ }^{4}$ Las instituciones cuyos fondos hacían presumir una alta probabilidad de hallar fragmentos, no han resultado fructíferas en este sentido, ya que a la variable temporal, también deben cruzarse otras, tales como la del lugar de origen del libro impreso y si los volúmenes han sido reencuadernados o sometidos a algún tipo de intervención, lo que lamentablemente suele traer aparejada la pérdida de los fragmentos alojados en la encuadernación original.

Otro aspecto problemático, al que hemos hecho mención, es el de la formación disciplinar específica que se requiere para analizar este tipo de corpus. Aunque nuestro país cuenta con una tradición en estudios de la Edad Media, hasta hace relativamente poco tiempo no se había promovido la enseñanza de paleografía y codicología en los niveles de grado y posgrado. Esto explica que, en décadas anteriores, salvo excepción, se le hubiese concedido escaso interés al estudio de los manuscritos medievales y humanísticos, ya sean completos ofragmentarios, que se conservan en Argentina. ${ }^{5}$ A esta cuestión, además, se suma la falta de financiamiento para emprender proyectos de investigación de manera sistemática, de modo que los resultados en general se ven limitados por la falta de recursos. En el estudio de los fragmentos esto se manifiesta tanto en las posibilidades de movilidad para acudir a las instituciones, la obtención de imágenes digitales de acuerdo con parámetros específicos para el ulterior análisis de las piezas y su publicación, así como la disponibilidad de diversas herramientas de investigación como son las bases de datos que agilizan la identificación de los textos, que obliga a acudir a otros medios, ya ampliamente superados, pero que son de acceso libre y gratuito. 
Esto último, si bien no siempre supone por consecuencia que no se pueda identificar el texto que porta cada fragmento, indefectiblemente implica que la tarea se torne mucho más lenta y laboriosa.

Nuestro proyecto comenzó formalmente a comienzos de 2020. De un elenco inicial de diecisiete instituciones, hemos explorado las colecciones de seis y reunido, además, cuatro fragmentos de coleccionistas privados. El aislamiento social preventivo y obligatorio nos ha obligado a suspender las visitas, de modo que las tareas que realizamos actualmente se basan en la descripción y el estudio del corpus que hemos establecido hasta la fecha.

\section{LOS FRAGMENTOS IN SITU}

A lo largo de toda la Edad Media y gran parte de la Edad Moderna, los manuscritos que quedaban obsoletos por distintos motivos fueron desmembrados y se les otorgó otra función por su versatilidad como soporte material, en especial el pergamino más que el papel. Así, entre sus múltiples destinos se cuenta su empleo asociado a la utilidad, ${ }^{6}$ por lo que no es infrecuente hallar partes de manuscritos en pergamino que alguna vez pertenecieron a un códice o un documento usados como material para confeccionar otras cosas, como sobres e indumentaria (Iglesias-Fonseca, 2019), o para las encuadernaciones de libros. No es nuestro propósito extendernos sobre los motivos de la obsolescencia de los manuscritos, pues estos pueden responder a una amplia variedad de razones de origen accidental o voluntario. ${ }^{7}$ Algunos manuscritos sufrían pérdidas de folios o cuadernillos por el deterioro de la encuadernación debido al uso y se consideraban material a descart ar; los textos litúrgicos, que constituyen una buena parte de los fragmentos manuscritos que sobreviven, fueron los más difundidos y los que tuvieron una destrucción sistemática por las modificaciones sucesivas hechas a las prácticas rituales (Albiero, 2018, p. 96). En los países en los que se impuso la Reforma, se desecharon los libros religiosos, que fueron mutilados y reutilizados como material para los archivos administrativos, tal como fue el caso de Suecia o Finlandia entre los años 1540 y 1630 (Ommundsen, 2008). Estas prácticas se intensificaron a partir del siglo XV -y en particular durante los siglos XVI y XVII- con la invención de la imprenta, ya que muchas de las obras que circulaban de manera manuscrita fueron reemplazadas por sus versiones impresas, en especial las teológicas, las filosóficas y las de derecho que formaban parte del currículo universitario. El alto costo del pergamino hacía que no se descartara, sino que se "reciclara" y que incluso, como es el caso de Barcelona, se produjese la convergencia de libreros y fabricantes de indumentaria en el mismo espacio de la ciudad, ambos dedicados a la compra y venta de libros (Iglesias-Fonseca, 2019, pp. 258-259).

Los fragmentos que hemos hallado pueden agruparse en dos clases diferentes: los folios o bifolios que han sido separados de su unidad codicológica original, y los fragmentos in situ que han sido reciclados y utilizados como refuerzo de encuadernación. Este último es el tipo más frecuente que se encuentra en las colecciones que hemos explorado: 39, sobre un total de 45 fragmentos que hemos identificado hasta el día de hoy, se hallan in situ.

Los fragmentos que aún están alojados en las encuadernaciones han captado comparativamente menos atención que los sueltos (Erwin, 2016, p. 189). Las causas de ello son múltiples. En aquellos casos en que existen registros catalográficos de las colecciones antiguas, la presencia de fragmentos muchas veces no es consignada ni descrita, lo que implica que permanezcan desconocidos hasta que una exploración manual de la encuadernación de cada libro los desvele. A su vez, a la dispersión -característica del fenómeno de la fragmentación en general- se suma el hecho de que los fragmentos in situ muchas veces tienen pequeñas dimensiones, lo que hace que resulte más difícil una investigación sistemática que ponga en relación partes de un mismo manuscrito presentes en diversos volúmenes. La creación de repositorios en línea contribuye a salvar esta dificultad, como el de Fragmentarium, ${ }^{8}$ que brinda acceso a estos testimonios manuscritos a través de reproducciones digitales y descripciones exhaustivas. 
Los fragmentos de este tipo son valiosos no sólo por las variantes que transmiten como testimonios de la tradición textual, sino también porque puestos en diálogo con el volumen que los hospeda, arrojan información respecto de la circulación de determinados textos en un momento y lugar específicos que muchas veces es posible recuperar únicamente a través de esta vía (Mullett, 2018). Aunque también en ocasiones, ya sea por la reencuadernación de los volúmenes, o por una diferente concepción en los criterios a seguir respecto de la conservación de los fragmentos manuscritos, puede ocurrir que éstos hoy se hallen separados del liber tradens, y se pierda de vista la conexión entre ambos. Es habitual encontrar en las bibliotecas europeas cajas con fragmentos separados de su contexto tras la restauración de los volúmenes que los alojaban.

El estado general de la encuadernación es fundamental a la hora de identificar la presencia de fragmentos in situ. Cuando ésta está en malas condiciones, no ha sido sometida a restauraciones y su estructura interna queda a la vista, estas pequeñas piezas de pergamino o de papel son fácilmente visibles. Otras veces, permanece en buen estado, pero aún sí se puede vislumbrar su presencia. Y también, dentro de los posibles escenarios, sucede que al deterioro de la encuadernación generado por diversas causas (el desgaste habitual, la acción de factores externos como la humedad o los insectos bibliófagos) le sigue el de los fragmentos. En algunas ocasiones, éstos llegan a despegarse y, en su lugar, por la transferencia de tinta del manuscrito, queda plasmada una imagen fantasma, que puede leerse en espejo.

\section{TiPología DE LOS FRAgMENTOS IN SITU POR SU UBICACión EN LOS VOLÚMENES}

Los fragmentos in situ pueden, a su vez, clasificarse según la función que cumplen en la encuadernación. Así, un tipo lo constituyen los que han sido utilizados como cubierta. Los folios y bifolios eran utilizados para forrar las tapas y lomos del impreso y para ello eran especialmente adecuados aquellos de gran tamaño, que con frecuencia pertenecían a manuscritos de obras litúrgicas (Reynhout, 2013, p. 104). Si el folio no era lo suficientemente amplio, se utilizaban dos superpuestos en el lomo, o bien pueden encontrarse uno como cubierta en la tapa frontal y otro en la trasera.

Este último es el caso de dos volúmenes que se encuentran en la Biblioteca y Centro de Investigación "San Alonso de Orozco" de la Orden de San Agustín. De los fondos con los que hemos trabajado, este es el de más reciente adquisición. En el año 2018, recibió una importante donación proveniente de la ciudad de Eindhoven (Holanda). Allí funcionaba la Biblioteca del Instituto Agustino (Bibliotheek Augustijns Insitituut) -anteriormente denominada Biblioteca Científica Mariënhage (Wetenschappelijke Bibliotheek Marinhage)- que formaba parte del Convento de Marinhage. ${ }^{9}$ La biblioteca había reunido en 1970 los fondos de otros conventos agustinianos de los Países Bajos, convirtiéndose así en una de las colecciones más importantes de la zona sur neerlandesa. En el año 2017, la institución se vio forzada a cesar sus actividades, por lo que una parte de sus fondos fue entregada a la Universidad de Tilburg, ${ }^{10}$ mientras que otra parte tuvo como destino la ciudad de Buenos Aires. Si bien aún no ha sido catalogada en su totalidad, contamos con un inventario de la donación en el que se registran 528 impresos comprendidos entre los siglos XV y XVII.

Los libros que presentan los folios de manuscritos adheridos a las tapas son los tomos primero y tercero de los cuatro de las Disputationes de controversiis christianae fidei adversus huius temporis haereticos del jesuita Roberto Bellarmino, impresos en 1615 en Colonia por Johann Gymnich y Anton Hierat, que portan las signaturas de la Bibliotheek Augustijns Insitituut M. Fol. 3. 1 y M. Fol. 3.2 respectivamente, con las que se los identifica en el inventario de la donación:

DISPVTATIOVM | ROBERTI | BELLARMINI | POLITIANI, | E SOCIETATE IESV, | S. R. E. CARDINALIS; | DE CONTROVERSIIS CHRISTIANAE | FIDEI, ADVERSVS HVIVS TEMPORIS | HAERETICOS, | QVATVOR TOMIS COMPREHENSARVM, | TOMUS PRIMVS. [...] COLONIAE AGRIPPINAE, | Sumptibus Ioannis Gymnici \& Antonij Hierat. | Anno M. DC. XV.| Superior. Permissu, et cum grat. et Priuil. S. Cas. Maeist. 
DISPVTATIOVM | ROBERTI | BELLARMINI | POLITIANI, | E SOCIETATE IESV, | S. R. E. CARDINALIS; | DE CONTROVERSIIS | CHRISTIANAE FIDEI, | ADVERSVS HVIVS TEMPORIS | HAERETICOS, | TOMUS TERTIVS. [...] COLONIAE AGRIPPINAE, | Sumptibus Ioannis Gymnici \& Antonij Hierat, | Anno M. DC. XV. | Superior, permissu, et cum grat. et Priuil. S. Cas. Maeist.

FIGURA 1

Biblioteca y Centro de Investigación “San Alonso de Orozco”, M. Fol. 3. 1 y M. Fol. 3. 2.
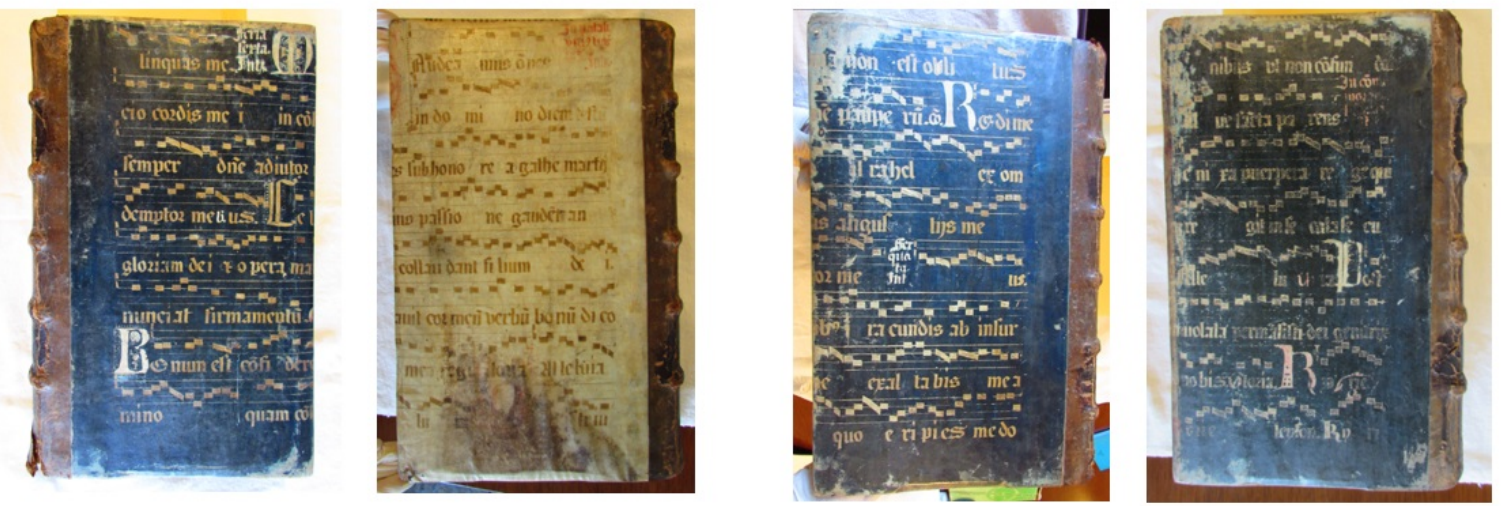

Fuente: fotografías propias con autorización de la institución.

En ambos tomos se ha utilizado un folio manuscrito para cubrir cada una de las tapas. El pergamino fue teñido de negro probablemente con el objeto de otorgarle un color uniforme, salvo por el folio de la tapa posterior de M. Fol. 3. 1. Esta tintura negra, sin embargo, no tuvo una buena adherencia sobre el texto que se buscó ocultar, dando por resultado una suerte de efecto "negativo", por el cual ésta resulta claramente legible. Por la interacción de esos diversos pigmentos, la escritura principal presenta un color parduzco, mientras que las tres iniciales "M", "L" y "B" y las rúbricas se ven ahora en un color blanquecino. El folio que no fue sometido a la tintura negra muestra una inicial " $G$ " en tinta roja con decoración en filigrana y un título rubricado.

El folio de la tapa anterior de M. Fol. 3.1 presenta fragmentos de piezas del propio de las misas del jueves y del viernes de la cuarta semana de Cuaresma, mientras que el de la posterior, el introito Gaudeamus omnes in Domino para la misa de la festividad de Santa Ágata ( 5 de febrero). El folio que cubre la tapa anterior de M. Fol. 3. 2 transmite el ofertorio, la comunión y el introito del propio de las misas del martes y miércoles de la quinta semana de Pentecostés y el de la posterior, piezas correspondientes a las misas del común de las Vírgenes Mártires y del común de la Virgen María.

El tipo de escritura es letra gótica septentrional, según se evidencia en el uso del signo tironiano cruzado para la abreviatura de "et", según la clasificación de Derolez (2003, p. 100) y la notación cuadrada sobre tetragrama corresponde a la variante empleada entre los siglos XV y XVII. ${ }^{12}$ Por sus características gráficas, estos fragmentos pertenecerían a un mismo codex discissus junto con los folios del volumen identificado con la signatura M. Fol. 3.1.

Otro lugar de la encuadernación donde es habitual encontrar fragmentos manuscritos es en las contratapas como folios de guarda adheridos. Para evitar que se soltaran los bordes de la cubierta doblados y pegados del lado de la contratapa, y para cubrir las partes del material que servía como tapa, se pegaba un folio de pergamino virgen o un folio manuscrito recortado en un tamaño apenas menor (Lanoë, 2010; Reynhout, 2013). En la mayor parte de las oportunidades, lo mismo que en el caso que analizábamos anteriormente, puesto que los fragmentos están pegados, sólo queda visible una de sus caras. Otras veces, cuando se ha desprendido, puede llegar a verse total o parcialmente el texto del vuelto.

Hemos encontrado un fragmento de este tipo en la encuadernación de un manuscrito litúrgico perteneciente a la colección de la donación Ferrer Vieyra de la Biblioteca Mayor de la Universidad Nacional de Córdoba. Además de poseer un importante fondo de origen jesuítico, la biblioteca ha recibido varias 
donaciones de particulares. Entre ellas, la del abogado, profesor y diplomático Enrique Ferrer Vieyra († 2008), quien en el año 2001 cedió la colección de manuscritos, incunables e impresos antiguos que había adquirido a lo largo de su vida. El volumen anfitrión está identificado con el número de inventario 385 . Se trata de un antifonario español de grandes dimensiones $(571 \times 379 \mathrm{~mm})$, encargado en 1609 por la monja Doña Ysabel de Mayorga para el Monasterio de Santa María la Real de Nájera, ${ }^{13}$ y ulteriormente renovado por Doña Vicenta del Spiritu Sancto en 1734. ${ }^{14}$

\section{FIGURA 2}

Biblioteca Mayor de la Universidad Nacional de Córdoba, Donación Enrique Ferrer Vieyra, N³85. Detalles de los ff. 1r-2r.
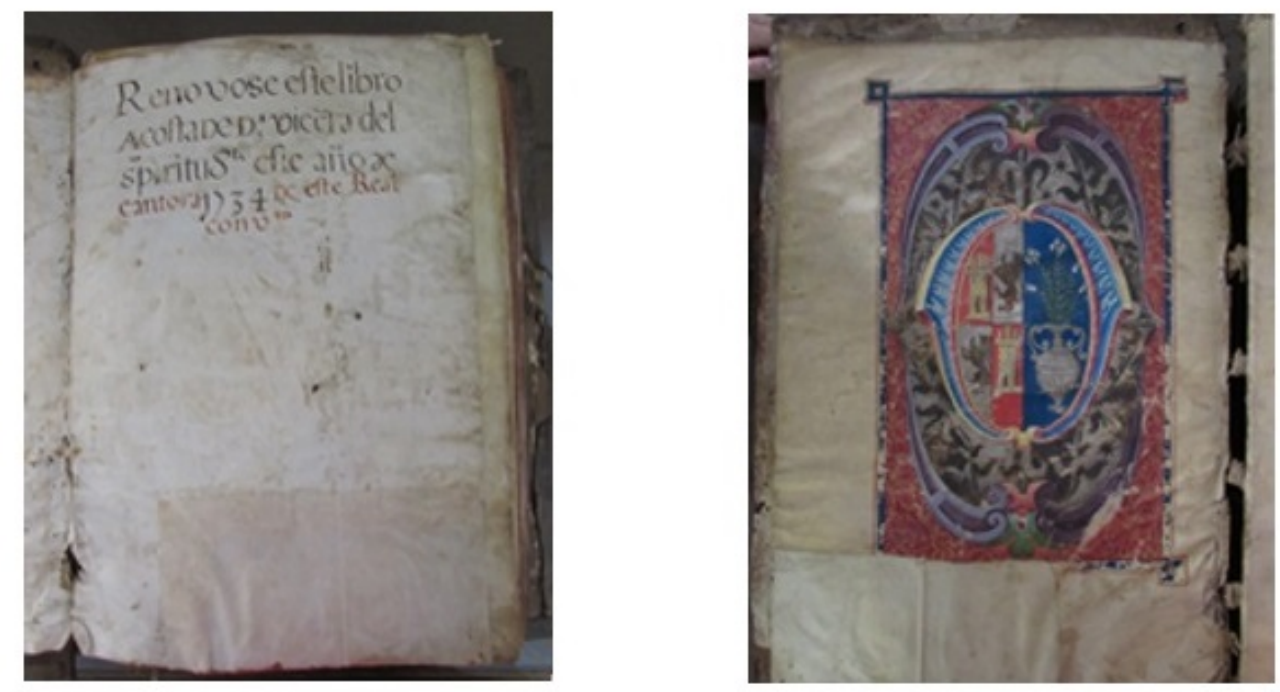

Biblioteca Mayor de la Universidad Nacional de Córdoba, Donación Enrique Ferrer Vieyra, № 385. Detalles de folio de guarda adherido a contra tapa frontal y folio de guarda volante recto y verso.
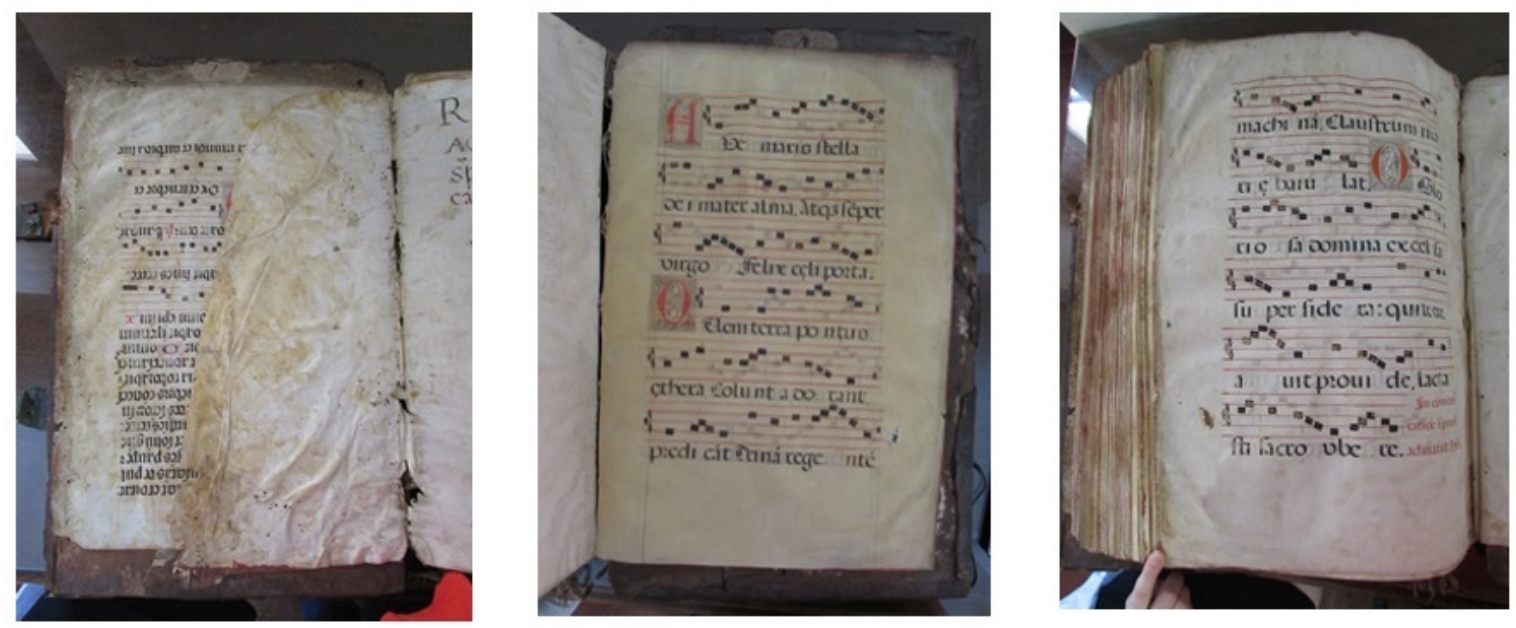

Fuente: fotografías propias con autorización de la institución.

En la contratapa anterior hay adherida una hoja de guarda virgen, que forma el bifolio con el folio $1 \mathrm{r}$ en el que consta la inscripción de la renovación del antifonario. Dicha hoja de guarda pegada no está completa, sino que ocupa de manera irregular la mitad de la contratapa, dejando a la vista un fragmento manuscrito 
que estaba adherido en primer lugar. El folio fue pegado de manera tal que quedó invertida la orientación del texto en relación con el resto del manuscrito. Es un fragmento de un códice litúrgico, escrito en una letra gótica septentrional, que podría datarse entre los siglos XV y XVI. La notación musical es gótica cuadrada sobre un pentagrama en tinta roja.

En la parte posterior del mismo antifonario se halla otro fragmento litúrgico, esta vez funcionando como un folio de guarda volante. Los folios de guarda de este tipo servían como protección del cuerpo de la obra contra la fricción producida por la manipulación del volumen, los efectos de la luz, los depósitos de polvo, las manchas y los distintos factores que podían potencialmente dañar el texto principal (Lanöe, 2010, p. 239). Estos fragmentos son aquellos que representan la situación "más feliz" (Reynhout, 2013, p. 105), pues resultan legibles en ambas caras y usualmente no están recortados de manera que se produzcan pérdidas. Sin embargo, al funcionar como protección del texto, tanto en este caso como en el anterior, están expuestos al desgaste derivado de su uso. En cuanto al fragmento que nos ocupa, el estado de conservación es muy bueno. Se trata nuevamente de un fragmento litúrgico, escrito en una letra gótica rotunda o gótica meridional, con notación musical del tipo cuadrada y pentagrama en tinta roja. La letra y la disposición del pautado de la página son idénticas a los del antifonario español, pero el folio de guarda volante carece de los títulos rubricados en el margen superior que se observan en los del volumen anfitrión.

Como refuerzo del bloque del texto, se solían utilizar tiras de pergamino o papel que a veces sobrepasan los cuadernillos dejando pestañas que sobresalen apenas algunos centímetros o incluso unos pocos milímetros. Estas tiras podían ser una grande, dispuesta longitudinalmente, o bien, varias pequeñas colocadas de manera perpendicular en el lomo entre el cosido o los nervios que sujetan los cuadernillos. Su identificación puede resultar factible dependiendo del módulo de escritura y de si se conserva una cantidad mínimamente significativa de texto, pero a veces sucede que, en fragmentos de pequeñas dimensiones con un módulo de letras relativamente grande, sólo es posible distinguir algunas letras o palabras sueltas, por lo que la identificación resulta en extremo dificultosa, sino imposible.

Estas pestañas quedan a veces a la vista y otras ocultas en la estructura de la encuadernación. No obstante, en ocasiones, la presencia de estos fragmentos, aunque no resulten legibles, llega a advertirse. Este es el caso de un fragmento que encontramos en la Biblioteca Central "Prof. Augusto Raúl Cortazar" de la Facultad de Filosofía y Letras de la Universidad de Buenos Aires, que se halla en el volumen identificado con la signatura TES 1-1-6, un impreso de Leiden de 1597 de la obra geográfica y etnográfica de Jordandes (o Jordanes) acerca de los godos y otros textos de varios autores sobre el tema:

IORNANDES | Episcopus Rauennas | DE | GETARVM, siue GOTHORVM | Origine \& rebus gestis. | Isidori Chronicon Gothorum, Vanda- | lorum, Sueuorum, et Wisogothorum. | Procopii Fragmentum, De priscis sedibus | \& migrationibus Gothorum, Græcè \& Lat. [...] Lvgdvni Batavorvm, | Ex officina Plantiniana, | Apud Franciscum Raphelengium. | M.D.XCVII. 
FIGURA 3

Biblioteca Central "Prof. Augusto Raúl Cortazar" (FFyL - UBA).

Detalle de Tes 1-1-6 contratapa anterior y lomo.
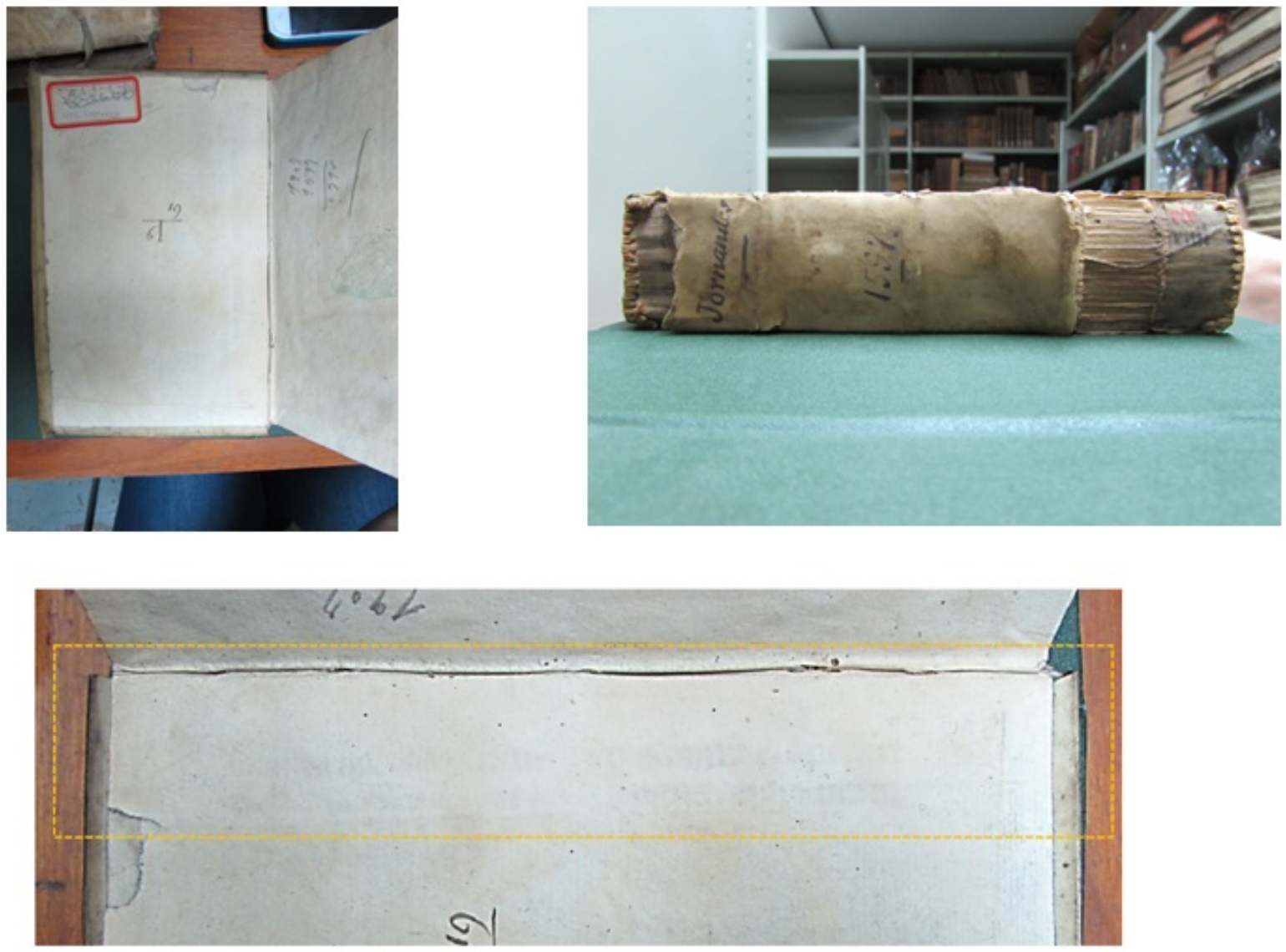

Fuente: fotografías propias con autorización de la institución.

Entre la contratapa y el folio de guarda adherido se puede entrever un fragmento longitudinal transparentado a través del papel. Para poder acceder a su lectura y análisis existen hoy técnicas no invasivas como el escaneo de macro fluorescencia de rayos X (MA-XRF según sus siglas en inglés) que permiten obtener imágenes y leer el texto (Duivenvoorden et al., 2017; Pouyet et al., 2017). Este mismo volumen tiene el lomo roto a la altura de las cabezadas superior e inferior, donde se observan dos tiras manuscritas en las costuras, pero su identificación no ha sido posible dado que sólo son visibles algunas letras.

Tal como hemos apuntado más arriba, la rotura de las encuadernaciones suele ser ventajosa para el estudio de los fragmentos in situ, pues deja al descubierto la estructura interna y los distintos elementos que la conforman. Este es el caso de dos tiras halladas en el volumen de 1614 del comentario del exégeta flamenco Cornelio a Lapide de la Biblioteca y Centro de Investigación "San Alonso de Orozco", K Fol. 23 A. L. 24 S:

COMMENTARIVS | IN | ESDRAM, NEHEMIAM, | TOBIAM, | IVDITH, ESTHER, | ET | MACHAB EOS, | AVCTORE | R. P. CORNELIO CORNELII | A LAPIDE | E SOCIETATE IESV, | Sacræ Scripturæ olim Louanij, posteà Romæ| Professore. | [...] | ANTVERPIÆ | Apud Ioannem \& Iacobvm Mevrsios. | ANNO M. DC. XIV. 
FIGURA 4

Biblioteca y Centro de Investigación "San Alonso de Orozco", K Fol. 23 A. L. 24 S.
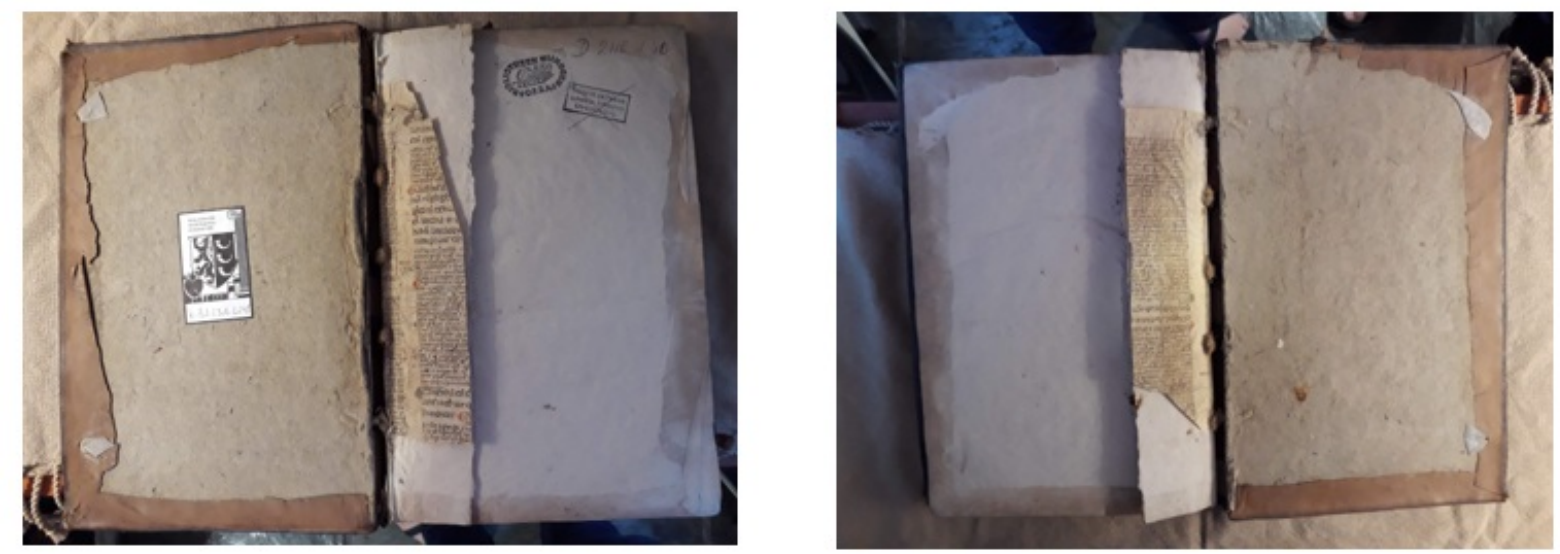

Fuente: fotografías propias con autorización de la institución.

Las dos tiras fueron utilizadas como refuerzos entre el folio de guarda y la contratapa anterior y posterior del volumen. A partir de la transcripción hemos logrado determinar que los fragmentos transmiten pasajes del Prognosticon de Hipócrates con comentarios atribuidos a Galeno en la traducción latina de Gerardo de Cremona, que circulaba en una compilación de obras médicas conocida como Articella. Desde el punto de vista paleográfico, se trata de un fragmento de un manuscrito universitario que sigue la estructura de glosa intercalada (Maniaci, 1996, p. 222 [M 434.10]), en la cual el texto principal comentado se copia en un módulo mayor que el de la glosa. El pautado del folio fue marcado con tinta a dos columnas. Las letras “.” de un solo compartimento, las "." finales realizadas en un único trazo con forma de "." elongada, el signo tironiano "et" cruzado y los tituli breves apuntarían a un tipo de escritura gótica septentrional, según la clasificación de Derolez (2003, p. 100), especialmente del tipo litterascholastica o textualiscurrens (también conocida en otras denominaciones paleográficas como semitextualis), tal vez parisina del siglo XIV. En los márgenes pueden observarse algunas glosas, una manicula y el dibujo de una cabeza sacando la lengua, intervenciones frecuentes en los manuscritos universitarios, en los que los estudiantes plasmaban anotaciones, signos y garabatos.

En otro volumen de la Biblioteca y Centro de Investigación "San Alonso de Orozco", identificado en el inventario de la donación con la signatura K fol. 39B. G 15. 1, hemos encontrado fragmentos utilizados como refuerzo de las costuras de cabezada:

EVANGELICVS | CONCIONATOR, | ET | NOVI HOMINIS | INSTITVTIO | EX DOCTRINA VERBI | Dei: Patris in veteri Testamento, | Filij in Euangelio, | Spiritus sancti in Paulo, Cano- $\mid$ nicis \& Apocalypsi [...] Auctore | Francisco Garcia del Valle | Calaguritano, è Soc. Iesv [...] LVGDVNI. | IACOBI CARDON ET PETRI CAVELLAT | M. D C. XXII 
FIGURA 5

Biblioteca y Centro de Investigación "San Alonso de Orozco", K fol. 39 B. G 15. 1.
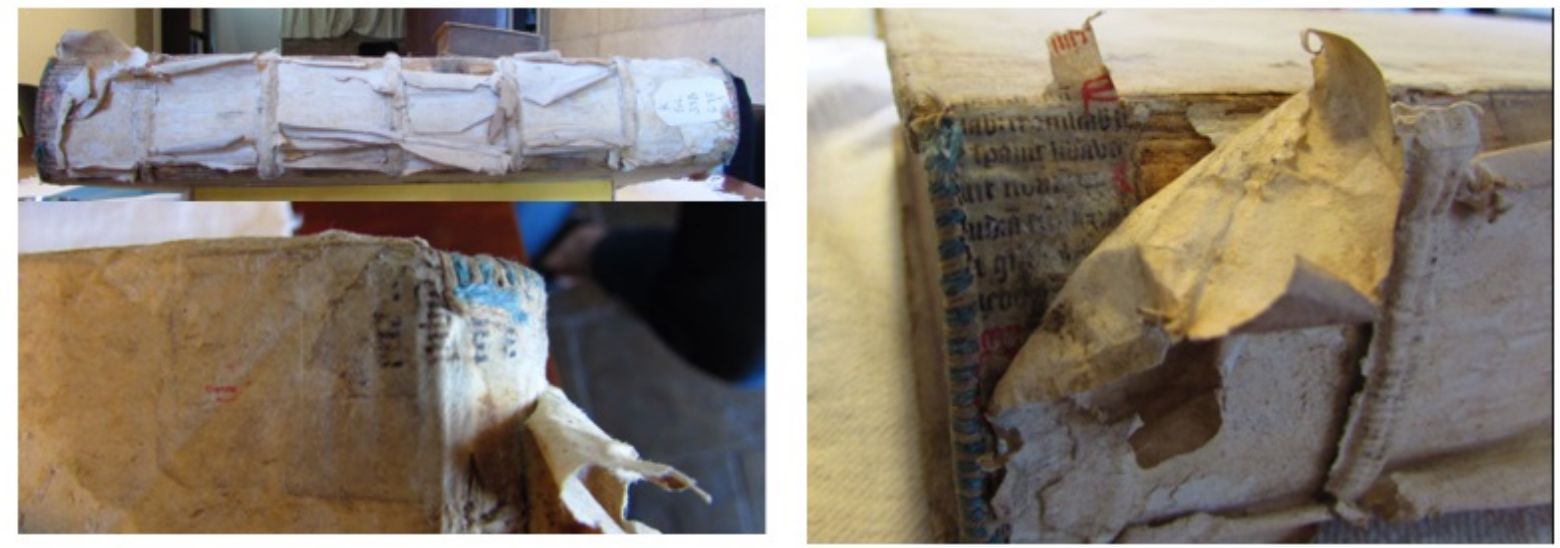

Fuente: fotografías propias con autorización de la institución.

Se trata de un texto litúrgico, escrito en letra gótica septentrional, de pequeño tamaño, con mayúsculas y títulos rubricados, posiblemente datada entre los siglos XIV y XV. El texto que alcanza a leerse se corresponde con los versículos 13 a 15 del Salmo 91, sin embargo, no resulta suficiente como para identificar con mayor precisión de qué tipo de texto litúrgico en concreto se trata.

En los casos en que la parte del lomo se ha separado por entero de la encuadernación, las tiras de refuerzo quedan expuestas. Un ejemplar de los discursos de Arístides de la Biblioteca Central "Prof. Augusto Raúl Cortazar" identificado con la signatura TES 1-3-18 constituye un ejemplo de este tipo, en el que pueden observarse fragmentos manuscritos que funcionan como refuerzos del lomo. Se trata de un impreso florentino de 1517:

\section{$\Lambda$ OГOI API $\Sigma$ TEI $\Delta$ OY. | ORATIONES ARISTIDIS.}

Colofón: Impressum bonis auibus optatam contigit metam hoc Aristidis opus | die xx. Maii. M. D. XVII. Florentiae sumptibus | nobilis uiri Philippi iuntae bibliopolae | Leonis. X. pontificis nostri | anno quinto.

FIGURA 6

Biblioteca Central "Prof. Augusto Raúl Cortazar” (FFyL - UBA), TES 1-3-18.
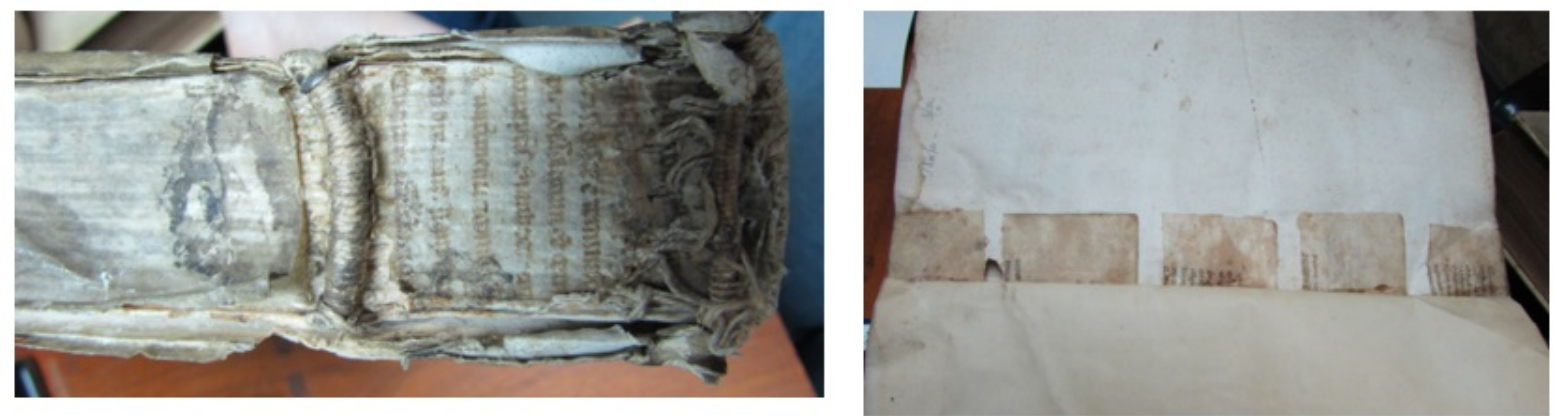

Fuente: fotografías propias con autorización de la institución.

Se observan cinco tiras que transmiten algunos pasajes de los capítulos IV y V de la Aristotelistopicorum libri interpretatio de Boecio. Están escritos en una letra gótica textual septentrional del siglo XIV y llegan a verse dos calderones en tinta azul y roja.

Muchas veces el deterioro es tal que, a pesar de que el fragmento quedó expuesto, éste también sufrió la acción de factores externos al punto de que su legibilidad es nula. Este es el caso de un impreso de 1553 que 
también se encuentra en la Sala del Tesoro de la Biblioteca Central "Prof. Augusto Raúl Cortazar", TES 1-3-17, que forma parte de la donación de Aldo Mieli: ${ }^{15}$

C. PLINII | SECVNDI HISTO- | RIAE MVNDI LIBRI XXXVII. | MAIORE, QVAM HACTENVS VN- | QVAM, STVDIO, FIDE, RELI- | GIONE EMENDATI. [...] LVGDVNI, | APVD IOANNEM | FRELLONIVM, | M. D. LIII. | Cum privilegio Regis.

FIGURA 7

Biblioteca Central “Prof. Augusto Raúl Cortazar” (FFyL-UBA), TES 1-3-17.

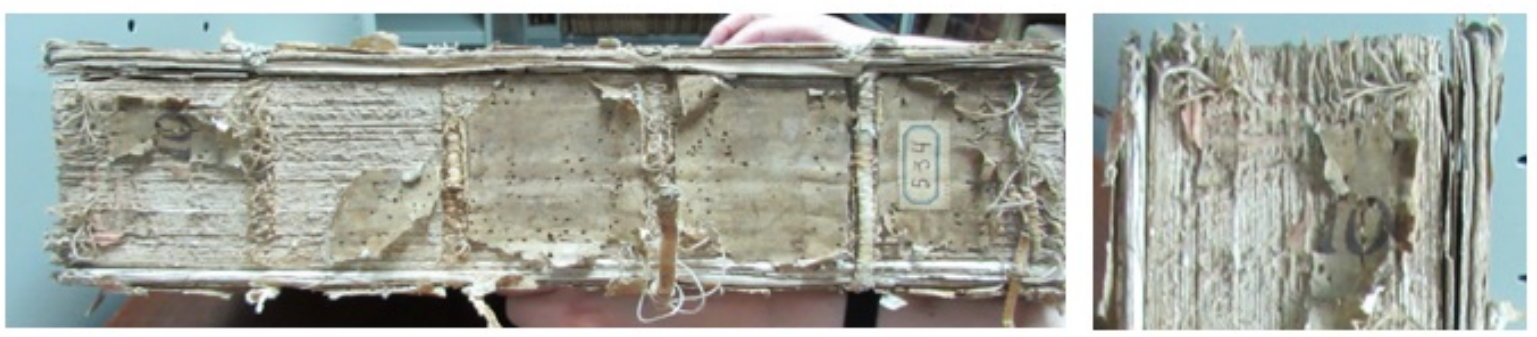

Fuente: fotografías propias con autorización de la institución.

El volumen tiene el lomo notablemente desgastado al punto que el cuero que lo cubría se deterioró, dejando al descubierto un fragmento manuscrito que, al estar expuesto, también fue víctima de insectos bibliófagos y, en su estado actual, resulta ilegible salvo por una letra "a" y restos de una rúbrica.

Es posible también encontrar que fragmentos que estaban adheridos y ya no ocupan su lugar original, han dejado una imagen "espejo" o "fantasma" (Pellegrin, 1980, p. 71; Reynhout, 2013, p. 105). Hemos encontrado un ejemplo en dos tiras de refuerzo de lomo de un impreso de la Biblioteca y Centro de Investigación "San Alonso de Orozco". El volumen anfitrión, impreso en Colonia en 1629, está inventariado bajo el número K 43B B50:

CONCIONES | siue | CONCEPTVS | THEOLOGICI AC | Praedicabiles | R. D. PETRI BESSAEISS | Theol. Doct. Reg. Galliar. Oratoris. | EDITIO POSTREMA AUCTIOR | Renouata Indice quadruplici, et | TOMIS IV. distributa | COLONIAE AGRIPPINAE | Apud IOANNEM KINCHIVM | sub Monocerote A. MDCXXIX | Cum priuil: S. Caes: Maj: speciali.

FIGURA 8

Biblioteca y Centro de Investigación "San Alonso de Orozco”, K 43B B50.
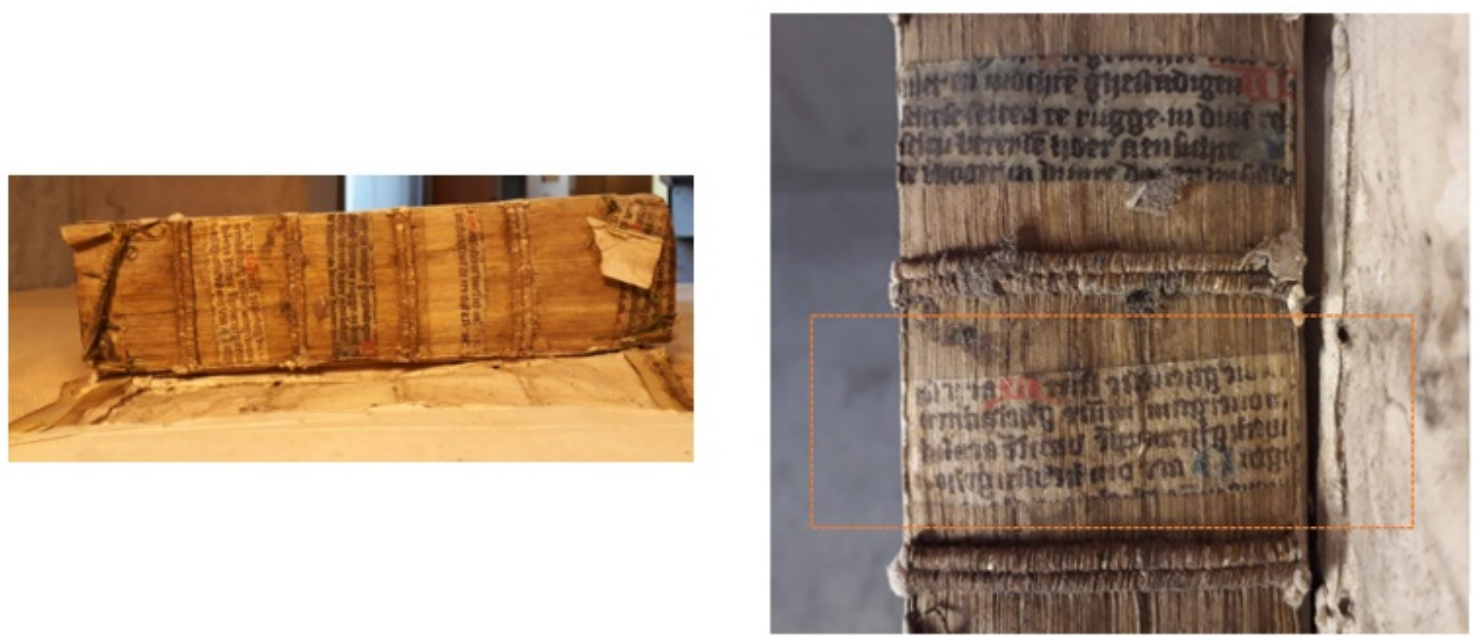

Fuente: fotografías propias con autorización de la institución. 
En el lomo que quedó al descubierto hay cuatro tiras de pergamino que transmiten una traducción de la devotio moderna de los Salmos en neerlandés medio, ${ }^{16}$ datada en el siglo XV y escrita en una letra gótica textual septentrional, con iniciales que alternan tinta roja y azul al inicio de cada verso. De las cuatro tiras, dos se han perdido y han dejado en su lugar una imagen en espejo. Su identificación ha sido posible utilizando herramientas digitales básicas que permitieron invertir la imagen.

\section{Conclusiones}

El propósito de este trabajo ha sido examinar algunas cuestiones fundamentales de la investigación sobre fragmentos manuscritos que llevamos adelante, y de los desafíos que esta supone, en particular, en Argentina. La dispersión territorial de los fondos implicó que, en una primera instancia, acotáramos el alcance geográfico de nuestra investigación, con la perspectiva de ampliar y añadir zonas nuevas posteriormente. El acceso a información precisa acerca de los fondos albergados por las instituciones es, además, una de las principales dificultades que hemos enfrentado. La elaboración de un catálogo que unifique el patrimonio bibliográfico antiguo en las distintas colecciones de nuestro país es un proceso largo y laborioso que supone la colaboración de diversos especialistas que complementen sus saberes acerca de la multiplicidad que el libro antiguo ofrece.

El estudio de fragmentos en Argentina ha sido hasta ahora un área de vacancia que nuestro proyecto busca subsanar enfrentando la consabida dificultad de conseguir financiamiento para la investigación. Nuestro país cuenta con un patrimonio histórico aún no conocido que requiere de nuestra atención, para que sea investigado y comunicado a la comunidad. Una aproximación desde el campo disciplinar de la fragmentología puede aportar información sobre un aspecto que se vincula directamente con el libro antiguo, pero que hasta ahora no ha recibido un estudio sistemático: la presencia de partes de manuscritos medievales y humanísticos alojados en las encuadernaciones.

\section{Agradecimientos}

No quisiéramos dejar de mencionar a quienes en el contexto de aislamiento de los últimos meses han tenido la gentileza de brindarnos su ayuda, ya sea respondiendo a nuestras consultas o haciéndonos llegar material bibliográfico para realizar este trabajo. Nuestra gratitud más sincera a William Duba (Université de Fribourg), Antoni Biosca Bas (Universitat d'Alacant), Peter Alexander Kerkhof (Leiden University) y Pablo Massa (Universidad de Buenos Aires).

Agradecemos a la Biblioteca Mayor de la Universidad Nacional de Córdoba, la Biblioteca y Centro de Investigación "San Alonso de Orozco" de la O. S. A. y la Biblioteca Central "Prof. Augusto Raúl Cortazar" de la Facultad de Filosofía y Letras de la Universidad de Buenos Aires por permitirnos trabajar sobre sus valiosos fondos.

\section{REFERENCIAS}

Albiero, L. (2018). I Frammenti liturgici dell'Archivio di Stato di Pavia. En Annuario dell'Archivio di Stato di Milano (pp. 95-130). Milán: Archivio di Stato di Milano.

Bamford, H. (2018). Cultures of the fragment. Uses of the Iberian manuscript, 1100-1600. Toronto-Buffalo-London: University of Toronto Press.

Cantera Montenegro, M. (2005). La ciudad de Nájera en tiempos del rey García. En J. I. de la Iglesia Duarte (Ed.). Garcia Sánchez III "el de Nájera" un rey y un reino en la Europa del siglo XI: XV Semana de Estudios Medievales, 
Nájera, Tricio y San Millán de la Cogolla del 2 al 6 de agosto de 2004 (pp. 39-72). España: Instituto de Estudios Riojanos.

Casazza, R. y Duba, W. (2018). Fragmento de una gramática medieval. Cuaderno de la BN, 3 (14), 12-13.

Derolez, A. (2003). The palaeography of gothic manuscript books: from the twelfth to the early sixteenth century. Cambridge: Cambridge University Press.

Duivenvoorden, J.; R., Käyhkö, A.; Kwakkel, E.; y Dik, J. (2017). Hidden Library. Visualizing fragments of medieval manuscripts in early-modern bookbindings with mobile macro-XRF scanner. Heritage science, 5(6). https://do i.org/10.1186/s40494-017-0117-6

Erwin, M. (2016). Fragments of medieval manuscripts in printed books: crowdsourccing and cataloging medieval manuscript waste in the book collection of the Harry Ransom Center. Manuscripta, 60(2), 188-247.

Fernández Rojo, A. (2009). Catálogo Nacional Unificado: Catálogo colectivo de libros antiguos existentes en Argentina en la Biblioteca Nacional de la República Argentina. En Experiencias en la organización y tratamiento de la información en las bibliotecas argentinas (vol. 1, pp. 287-300). Buenos Aires: Biblioteca Nacional de la República Argentina.

Iglesias-Fonseca, J. A. (2019). 'Instruments inùtils o no importants per lo monastir', en los márgenes de la codicología fragmentos y membra disiecta. En G. Avenoza, L. Fernández Fernández y L. Soriano Robles (Coords.). La producción del libro en la Edad Media: una visión interdisciplinar (pp. 247-291). Madrid: Sílex.

Lanoë, G. (2010). La protection de l'écrit. Les gardes approche d'une typologie. En Matériaux du livre médiéval: Actes du colloque du Groupement de recherche (GDR) 2836 "Matériaux du livre médiéval", Paris, CNRS, 7-8 novembre 2007 (pp. 237-248). Turnhout: Brepols.

Maniaci, M. (1996). Terminologia del libro manoscritto. Milano: Editrice Bibliografica.

Molinari, A.; Biondi, N. y Abate, E. (2019). Textus invisibilis. An integrated research approach to the study of the manuscript fragments preserved at the State Archive in Urbino. En G. Dall'Olio y S. Pivato (Eds.). Urbino in età moderna e contemporanea (pp. 215-260). Rimini: Panozzo.

Mullett, R. (2018). In-situ manuscript fragments in the incunables of the Bodleian Library, Oxford: a fragmentarium case study. Fragmentology, 1, 111-120.

Ommundsen, Ä. (2008). From books to bindings — and back. medieval manuscript fragments in Norway. Gazette du livre médiéval, 52-53, 34-44.

Parada, A. E. (2012). El libro antiguo y lo conjetural. Aproximaciones con vocación latinoamericana. En El dédalo y su ovillo. Ensayos sobre la palpitante cultura impresa en Argentina (pp. 31-59). Buenos Aires: Instituto de Investigaciones Bibliotecológicas, Facultad de Filosofía y Letras, Universidad de Buenos Aires.

Pellegrin, E. (1980). Fragments et membra disiecta. Codicologica, 3, 70-95.

Pouyet, E.; Devine, S.; Grafakos, T.; Kieckhefer, R.; Salvant, J.; Smieska, L., ..., Walton, M. (2017), Revealing the biography of a hidden medieval manuscript using synchrotron and conventional imaging techniques. Analytica chimica acta, (982), 20-30.

Reynhout, L. (2013). Défets manuscrits dans les imprimés anciens de la Bibliothèque royale de Belgique. In Monte Artium, 6, 97-120.

Robles Sierra, A. (1967). A propósito de un nuevo fragmento autógrafo de Santo Tomás de Aquino. Analecta sacra tarraconensia: revista de ciències historicoeclesiàstiques, 40(1), 65-76.

Sala, P. S.; Baratelli, M. y Burgos, M. C. (2007). La Biblioteca Central de la Facultad de Filosofía y Letras. Ejemplares valiosos del Tesoro. Espacios de critica y producción, 34, 86- 98.

Santos, D.; Ubierna, P. y Hernández, A. (2011). Informe sobre MNAD 1453. Buenos Aires. [Informe inédito incorporado al legajo correspondiente a la pieza del Museo de Arte Decorativo.]

Tesler, M. (2020). Un caso de lesa cultura en la Sociedad Hebraica Argentina. Buenos Aires: s.n. 


\section{SITIOS WEB}

Augustijns Instituut Utrecht (2009). Augustijns Instituut Utrecht. Augustijns Instituut Utrecht. Recuperado de http s://www.augustinus.nl/C90-Historie.html

Fragmentarium Digital Laboratory (2020). Fragmentarium, University of Fribourg. Recuperado de https://fragmen tarium.ms

Tilburg University (2001). Augustijnse collectie, Recuperado de https://www.tilburguniversity.edu/nl/campus/unive rsiteitsbibliotheek/collecties/augustijnse-collectie

\section{Notas}

1 Este trabajo se inscribe en el marco del proyecto de investigación "Medieval Manuscript Fragments in Argentina" auspiciado por Fragmentarium. Digital Research Laboratory for Medieval Manuscript Fragments y localmente radicado en el IIBICRIT-SECRIT/CONICET en el que participamos junto con la Dra. Valeria Buffon.

2 Este equipo está integrado localmente por las Dras. Soledad Bohdziewicz, Marcela Borelli, Valeria Buffon, Cecilia Rusconi, el Lic. Pablo Massa y los Dres. Fernando Ruchesi y Roberto Casazza.

3 En este trabajo utilizamos la expresión "libro antiguo" para referirnos a libros impresos desde el siglo XV hasta el XVII, arco temporal en el que mayormente se produjo la reutilización de fragmentos de manuscritos como insumo de encuadernación. Para una aproximación al concepto del libro antiguo desde la perspectiva de la bibliotecología latinoamericana remitimos a Parada (2012, pp. 31-59).

4 Este es el caso de diez libros impresos a principios del siglo XX que pertenecieron a la biblioteca personal de Victoria Ocampo.

5 No podemos dejar de mencionar como antecedente dos aportes sobre fragmentos manuscritos. En primer lugar, el informe redactado por Santos, Ubierna y Hernández sobre un fragmento del Museo Nacional de Arte Decorativo (2011) y el artículo de Casazza y Duba (2018) sobre el fragmento hallado en el "manuscrito encadenado" de la Biblioteca Nacional "Mariano Moreno".

6 La destrucción de códices responde no sólo a los fines prácticos que mencionamos someramente aquí, sino que también puede obedecer a motivaciones simbólicas y religiosas, como es el caso de los fragmentos de los Coranes del siglo XIV hallados en los cielorrasos del palacio de Aljafería en Zaragoza, que, en opinión de Bamford cumplen una función apotropaica con el fin de proteger a las personas del edificio (Bamford, 2018, pp. 113-117), o como ocurre, por caso, con el fragmento autógrafo de Santo Tomás de Aquino del monasterio de Santa Inés de Zaragoza, que se ha relacionado con el manuscrito napolitano Biblioteca Nazionale, I, B. 54, convertido en reliquia en el siglo XVIII (Robles Sierra, 1967).

7 Para una descripción exhaustiva sobre los motivos del desmembramiento de códices cf. Pellegrin (1980) e IglesiasFonseca (2019).

8 Cf. Fragmentarium. Laboratory for Medieval Manuscript Fragments.

9 Para una reseña histórica sobre la biblioteca y sus fondos puede consultarse la página del Augustijns Instituut de Utrecht.

10 Cf. Augustijnse collectie de la Tilburg University.

11 La signatura M. Fol. 3. 2 del tomo tercero deja inferir que la biblioteca no llegó a poseer los cuatro tomos en que fue impresa la obra.

12 Agradecemos al Lic. Pablo Massa por la elaboración de los informes de identificación de estos folios.

13 En el vuelto del primer folio hay un escudo dividido en dos mitades: la mitad izquierda contiene el escudo del Reino de Castilla y León con dos cuarteles con castillos de tres almenas y otros dos cuarteles con leones rampantes. La segunda mitad tiene una jarra con la inscripción "Ave Maria" y un ramo de azucenas, símbolo del monasterio de Nájera. La jarra con el ramo de azucenas está asociada a los relatos de su fundación (cf. Cantera Montenegro, 2005, p. 47). Detrás, se advierte el escudo de los dominicos, es decir, la cruz flor de lis de color blanco y negro.

14 En el folio 1r se deja constancia de la renovación del volumen por medio de la siguiente nota: "Renovose este libro | a costa de $\mathrm{D}^{\text {a }}$ Vicenta del Spiritu $S^{\text {to }}$ este año de $\mid 1734$ cantora de este Real $\mid$ conu ${ }^{\text {to" }}$.

15 Aldo Mieli (1879-1950) fue un reconocido epistemólogo italiano que emigró a Argentina trayendo consigo una rica biblioteca que legó a la Facultad de Filosofía y Letras de la Universidad de Buenos Aires (Sala, Baratelli y Burgos, 2007).

16 Agradecemos al Dr. Peter Alexander Kerkhof y al Dr. William Duba por su colaboración en la identificación de estos fragmentos. 